\title{
DECOMPOSITION AND RECOMPOSITION STRATEGIES OF PROFESSIONAL ENGINEERING DESIGN TEAMS
}

\author{
Milovanovic, Julie (1); \\ Gero, John (2); \\ Becker, Kurt (3) \\ 1: UMR AAU-CRENAU, Graduate School of Architecture of Nantes, Nantes, France; \\ 2: Department of Computer Science and School of Architecture, University of North Carolina at \\ Charlotte, Charlotte, USA; \\ 3: Department of Engineering Education, Utah State Univ
}

\begin{abstract}
Designers faced with complex design problems use decomposition strategies to tackle manageable sub-problems. Recomposition strategies aims at synthesizing sub-solutions into a unique design proposal. Design theory describes the design process as a combination of decomposition and recomposition strategies. In this paper, we explore dynamic patterns of decomposition and recomposition strategies of design teams. Data were collected from 9 teams of professional engineers. Using protocol analysis, we examined the dominance of decomposition and recomposition strategies over time and the correlations between each strategy and design processes such as analysis, synthesis, evaluation. We expected decomposition strategies to peak early in the design process and decay overtime. Instead, teams maintain decomposition and recomposition strategies consistently during the design process. We observed fast iteration of both strategies over a one hour-long design session. The research presented provides an empirical foundation to model the behaviour of professional engineering teams, and first insights to refine theoretical understanding of the use decomposition and recomposition strategies in design practice.
\end{abstract}

Keywords: Design cognition, Systems Engineering (SE), Design process, Protocol analysis, Problem decomposition

\section{Contact:}

Milovanovic, Julie

AAU-CRENAU

Graduate School of Architecture

France

julie.milovanovic@crenau.archi.fr 


\section{INTRODUCTION}

Designing is a unique and rich cognitive process (Cross, 1982; Lawson, 2006), and is often treated as a type of problem solving (Simon, 1969). Design problems have been described as ill-structured (Simon, 1973) or wicked (Rittel and Webber, 1973), therefore require designers to decompose (Alexander, 1964; Rowe, 1992), to frame (Akin and Akin, 1996), or to structure (Goel and Pirolli, 1992) design problems to progress in the design process. Theoretical design frameworks depict a first phase of problem decomposition, followed by the generation of sub-solutions that are later assembled, or recomposed, to obtain an overall design solution (Alexander, 1964; Pahl et al., 2007; Simon, 1969).

Empirical studies point toward a fuzzier approach to design decomposition (Ho, 2001; Liikkanen and Perttula, 2009) that is closer to design problem/solution co-evolution (Dorst and Cross, 2001; Maher and Poon, 1996), where formulating solutions helps designers reframe the problem in early design stages. Hence, the temporal dynamics of decomposition and recomposition processes are likely to follow an iterative pattern (alternation of decomposition and recomposition processes), more than a distinct phasing (decomposition then recomposition).

Insufficient knowledge is known about the temporal dynamic of decomposition and recomposition strategies or the relation between design processes (analysis, synthesis, evaluation) and decomposition/recomposition strategies. This paper begins to address that gap. Nine teams of three professional engineers from the same company in the USA were given the same design task: to design a future personal entertainment system. Results from analysing the sessions provide new insights on how teams of professional engineers rely on decomposition and recomposition strategies to advance in their design process.

\section{RELATED WORK}

\subsection{Engineering design processes}

The engineering design literature commonly describes the design process through a top-down approach. For instance, Pahl et al. (2007) delineate three phases in the design process: producing high level conceptual designs, generating a preliminary layout and detailing designs. Breadth-first or depth-first approaches to designing stem from a top-down decomposing approach. Breadth-first implies that several design options are explored in parallel before detailing one (Ball et al., 1997). Using this strategy, designers would formulate sub-goals at the same design level (sub-systems) before detailing solutions for one of the sub-goals. The depth-first approach relies on first developing a sub-goal before switching to another one. Empirical findings based on protocol studies point toward a mix of initial breadth-first strategies and occasional depth-first decomposition strategies in individual design (Goel and Pirolli, 1992; Ho, 2001; Liikkanen and Perttula, 2009; Sun et al., 2016) describing an opportunistic approach to design (Visser, 1994). Teams do not necessarily take a breadth-first approach, contrary to theory about design problem solving (Gralla et al., 2019). The lowest levels of design (preliminary layout and detailed design) are tackled simultaneously by design teams. The design teams observed by Gralla and colleagues (2019) adopted a very different decomposition strategy resulting in diverse sequences of moving between high level concept, preliminary layout and detailed design.

\subsection{Problem decomposition and recomposition strategies}

The concept of opportunistic design echoes the implicit decomposition processes observed by Ho (2001) and Guidon (1990). Implicit decomposition is related to designers' recognition of a solution or partial solution to a design problem (Liikkanen and Perttula, 2009), i.e., a system 1 thinking mode (Kahneman, 2011; Kannengiesser and Gero, 2019). On the other hand, explicit decomposition implies decomposing the problem upfront and corresponds to the theoretical definition of problem decomposition. Explicit problem decomposition relies on a controlled process (Liikkanen and Perttula, 2009) or system 2 thinking mode (Kahneman, 2011; Kannengiesser and Gero, 2019). Designers need to know and structure the problem beforehand, for them to decompose it. In their study, Liikkanen and Perttula (2009) found designers to rely on implicit decomposition more often than on explicit decomposition. When employed, explicit decomposition is usually preceded by implicit decomposition.

These empirical studies focus mainly on problem decomposition without tackling recomposition processes although this strategy is crucial for designers to generate solutions. In Gero and Song (2017) and Song et al. (2016), both types of design strategies are analysed. Findings reveal a balance between 
the use of decomposition and recomposition strategies for collaborative design sessions. Teams of professionals were found to rely more on decomposition/recomposition strategies in the first half of the session compared to the second half.

\section{RESEARCH QUESTIONS}

Most empirical studies tackling design thinking at a systems' level have: 1) focused on decomposition strategies, not on its dual strategy (decomposition/recomposition) (Gralla, 2019; Guindon, 1990; Ho, 2001), 2) are based on individuals, not teams (Ball et al., 1994; Ho, 2001) and 3) studied engineering students, not professionals (McCracken, 1997, Austin-Breneman et al., 2012). In this study, we articulate the behaviour of professional designer teams in terms of decomposition/recomposition strategies with design processes used. The research questions are:

- What are the decomposition and recomposition strategies of professional designers teams over time?

- How do decomposition and recomposition strategies correlate with design processes?

We expect designers to rely on decomposition strategies early in the design process and focus more on recomposition strategies later in the design session (Figure 1).

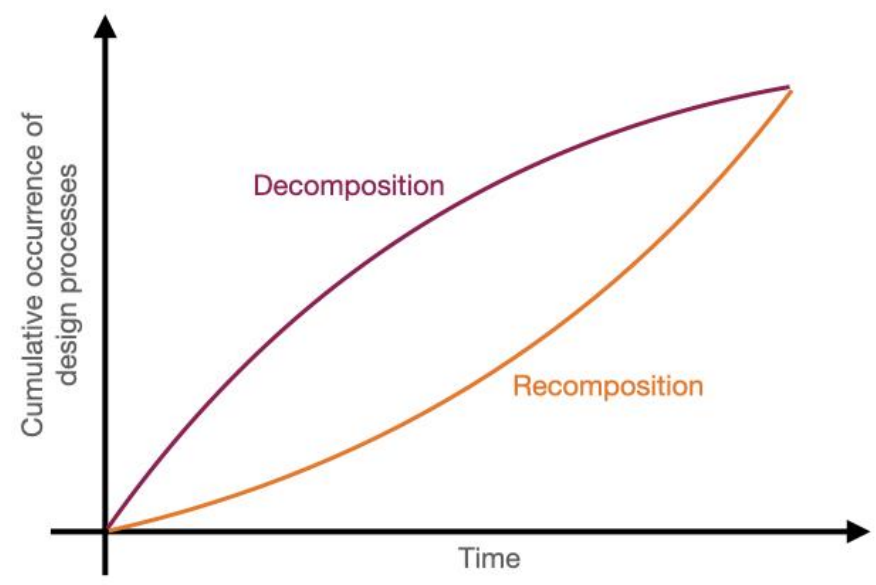

Figure 1. Prediction of temporal modelling of decomposition / recomposition strategies

\section{METHODOLOGY}

\subsection{The study}

In order to study decomposition and recomposition strategies in professional engineering teams an in vitro experiment was conducted with engineers, all from the same company. In total, 27 engineers working in the same company participated in a think-aloud protocol experiment and were grouped into teams of 3. This company specializes in developing solutions for automotive safety. All engineers were used to working together as the company uses a lean manufacturing and production process in their product development and production that requires them to engage on projects together. They were given 60 minutes to complete a design task. Teams were asked to design a future personal entertainment system (see brief in Appendix). Teams did not receive any instruction on using decomposition and recomposition design strategies, but teams were prompted to adopt systems designs as the design task specifically ask them to design a system. The background of each team member ranged from mechanical engineer, systems engineer, manufacturing engineer, product development engineer, safety engineer to electronics and technology engineer. All engineers were experts, meaning that they had more than 10,000 hours of professional design experience. Each codesign session was video recorded on the company's site for later analysis.

\subsection{Measuring design strategies and processes}

We analysed the data from the think-aloud protocols to measure how engineers decompose the design problem into sub-problems and recompose them as part of the process of determining their systems design strategy. In previous work, multiple levels of a design problem were used to analyse problem 
decomposition (Gero and McNeill, 1998; Ho, 2001; Song et al., 2016). We used the framework developed by Gero and McNeill (1998) which accounts for three levels of hierarchy describing the system. The highest level is the systems level where designers consider the problem as a whole. The second level is the sub-system level. The smallest scale of the system is a detail of one of the subsystems of the design problem. These levels map onto Pahl et al. (2007) design phases (conceptual designs, preliminary layout and detailing designs) used in decomposition studies (Gralla et al., 2019; Tobias et al., 2015).

The framework used to study design cognition is the Function-Behaviour-Structure (FBS) ontology (Gero, 1990; Gero and Kannengiesser, 2004). The same framework was used in Gero and Song (2017), Ho (2001) and Song et al. (2016). The FBS ontology describes design issues or elements about the object being designed: Requirement (R) includes the design brief and norms; Function (F) represents what the design object is for; expected Behaviour $(\mathrm{Be})$ denotes the design intentions in terms of how it is expected to behave; Structure (S) is defined as the elements and their relationships of the design object; Behaviour derived from structure (Bs) accounts for how the object behaves based on the proposed design Structure (S) and Description (D) is an external representation of the design object (Figure 2). The FBS ontology also accounts for design processes that are the transitions from one design issue to another. The eight design processes are: Formulation, Synthesis, Analysis, Evaluation, Documentation, Reformulation 1, Reformulation 2 and Reformulation 3 (Figure 2).

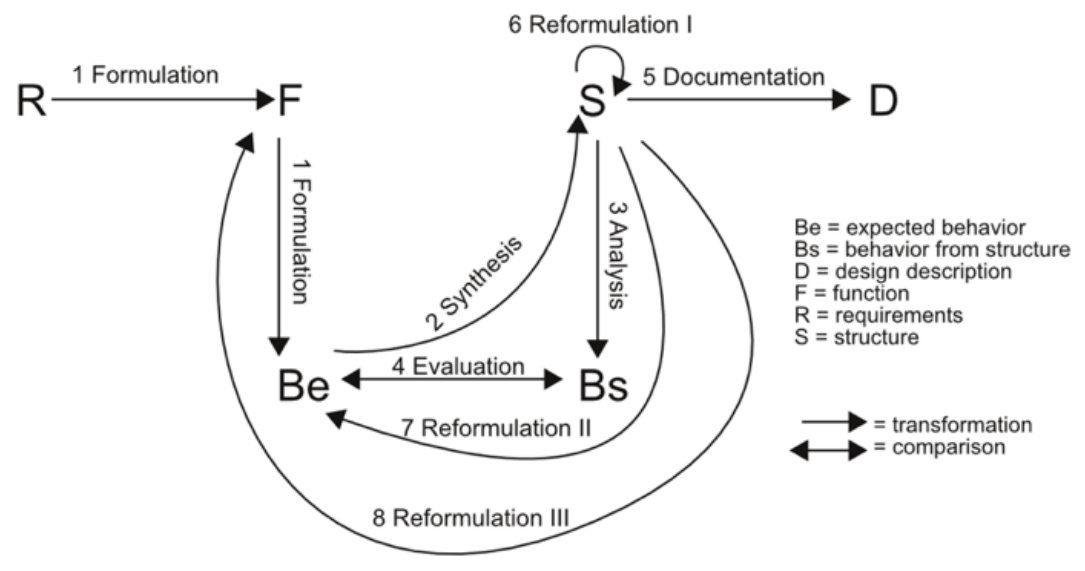

Figure 2. FBS framework showing design issues and design processes (based on Gero, 1990)

\subsection{Data analysis}

Protocol analysis is used to infer cognitive behaviour from the teams (Ericsson and Simon, 1984; Van Someren et al., 1994). Each co-design session is transcribed, segmented and coded. Segments represent a single design issue of the FBS ontology (R, F, Be, S, Bs or D) and were coded based on each segment's content. Protocols were also coded with the systems levels: level 1 is the whole system, level 2 for sub-systems and interactions between sub-systems and level 3 for details of a subsystem. For example, in the context of designing a future personal entertainment system, a tablet or phone would be coded as system level 1, an app on the tablet would be coded as system level 2 and a game within the app would be coded as system level 3. The FBS ontology description of the design activity can be mapped onto models of systems engineering like the INCOCE model (INCOSE, 2015), making it a suitable framework for the analysis of empirical data of engineers designing in systems (Kannegiesser \& Gero, in review).

Each of the nine protocols were coded by two researchers. In total, three trained researchers worked together in coding teams of two. Agreement between coders was measured with Cohen's kappa. It reached 0.79 for the FBS design issue codes and 0.74 for the systems level codes, which ensures the reliability of the coding.

Design processes are generated from the FBS design issue code. For instance, an expected behaviour (Be) followed by a structure (S) is a synthesis process. Decomposition and recomposition strategies are generated based on transitions from the systems' level codes. Decomposing the design problem implies formulating a system level design issue (level 1), followed by a sub-system design issue (level 2). A 
decomposition strategy is represented by a transition from system level 1 to 2 , system level 1 to 3 and system level 2 to 3 . A recomposition strategy is represented by a transition from system level 3 to 1 , system level 3 to 2 and system level 3 to 2 . No changes in systems level indicates that the team is engaging cognitive effort on one of the system levels. Examples are given in Table 1.

Table 1. Example of coding

\begin{tabular}{|l|c|c|c|c|}
\hline \multicolumn{1}{|c|}{ Utterance } & FBS code & Design process & $\begin{array}{c}\text { System } \\
\text { level }\end{array}$ & System transition \\
\hline It's got to be able to connect to & $\mathrm{Be}$ & - & 2 & - \\
\hline all the in-home, you know. & $\mathrm{S}$ & Synthesis & 2 & Same level \\
\hline $\begin{array}{l}\text { So, if you have a Wi-Fi. The Wi-Fi } \\
\text { stuff.. }\end{array}$ & $\mathrm{S}$ & Reformulation 1 & 3 & Decomposition \\
\hline or Bluetooth & $\mathrm{S}$ & Reformulation 1 & 3 & Same level \\
\hline or whatever features & $\mathrm{S}$ & Reformulation 1 & 3 & Same level \\
\hline $\begin{array}{l}\text { and be able to network with all of } \\
\text { them. }\end{array}$ & $\mathrm{Be}$ & Reformulation 2 & 3 & Same level \\
\hline $\begin{array}{l}\text { And then you got to have, you know, } \\
\text { with the TVs }\end{array}$ & $\mathrm{S}$ & Synthesis & 2 & Recomposition \\
\hline and then connecting & $\mathrm{Bs}$ & Analysis & 2 & Same level \\
\hline to your entertainment things. & $\mathrm{S}$ & Synthesis & 2 & Same level \\
\hline
\end{tabular}

\section{RESULTS}

\subsection{The distribution of decomposition and recomposition strategies is balanced}

The average distributions of design strategies across all nine sessions highlight a dominance of cognitive effort on remaining focused on one level. During the design process, recomposition (increasing system level) and decomposition (decreasing system levels) strategies are balanced and represent around $20 \%$ of the total number of transitions (Figure 3(a)). The distribution of decomposition strategies from the whole system to sub-systems $(1>2)$ is approximately equal to the distribution of decomposition strategies from sub-systems to sub-systems details (2>3) (Figure 3(b)). Recomposition strategies from system details to sub-systems $(3>2)$ is more frequent than recomposition strategies from sub-systems to the whole system $(2>1)$. The standard deviation across teams is low which accounts for a certain homogeneity in team behaviour.
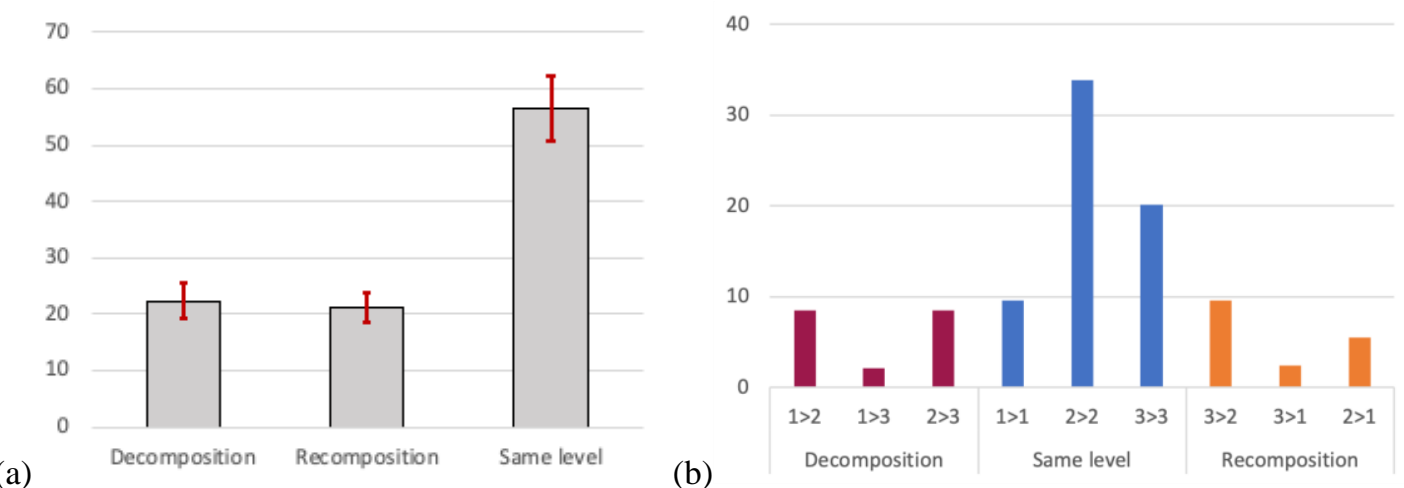

Figure 3. Average distribution in percent of (a) the decomposition / recomposition strategies for all teams, (b) detail for each decomposition / recomposition strategy

\subsection{Decomposition and recomposition strategies over time}

To analyse the temporal dynamic dimension of the teams' use of decomposition and recomposition strategies, we modelled the cumulative occurrence over time of decomposition, recomposition and same level transition strategies (Figures 4 and 5). The fit of same system level transitions occurrence over time is almost linear $\left(\mathrm{R}^{2}=0.92\right.$, see Figure 4$)$. For both decomposition and recomposition 
strategies, the model follows a linear fit (for the decomposition processes $\mathrm{R}^{2}=0.95$, for the recomposition processes $R^{2}=0.96$ ). The slope of both trend lines is similar which implies a similar frequency or occurrence for decomposing and recomposing strategies (Figure 4(a) and (b)). The slope of the same level transitions is more than twice as steep as the fit curves for the decomposition and recomposition strategies. In other words, design teams exploit all three types of strategies in a regular pattern over time. This is previously unknown design behavior.

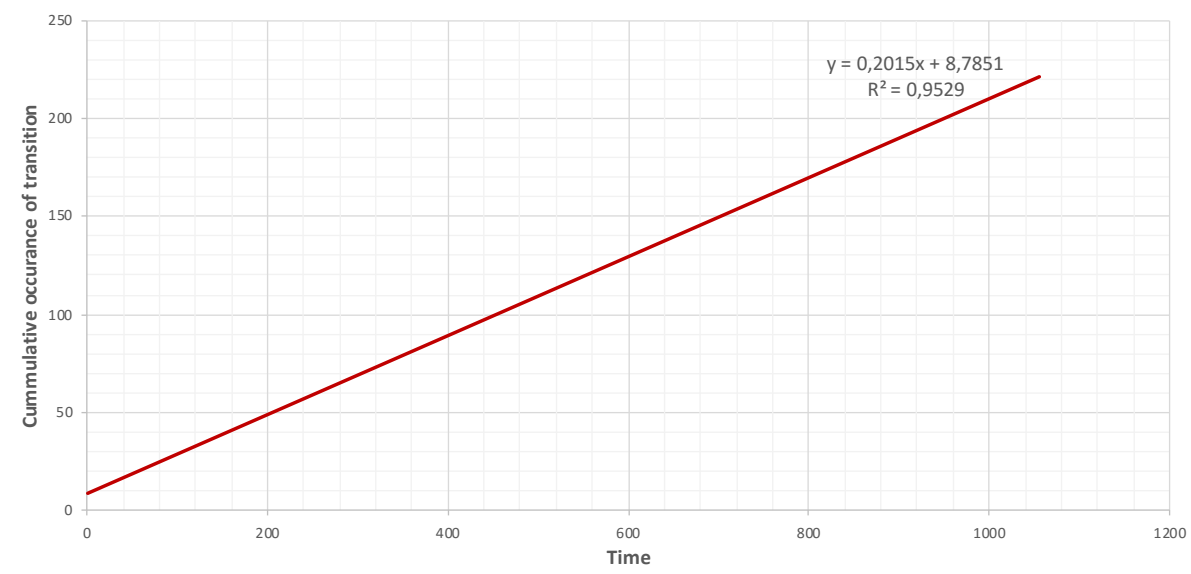

(a)
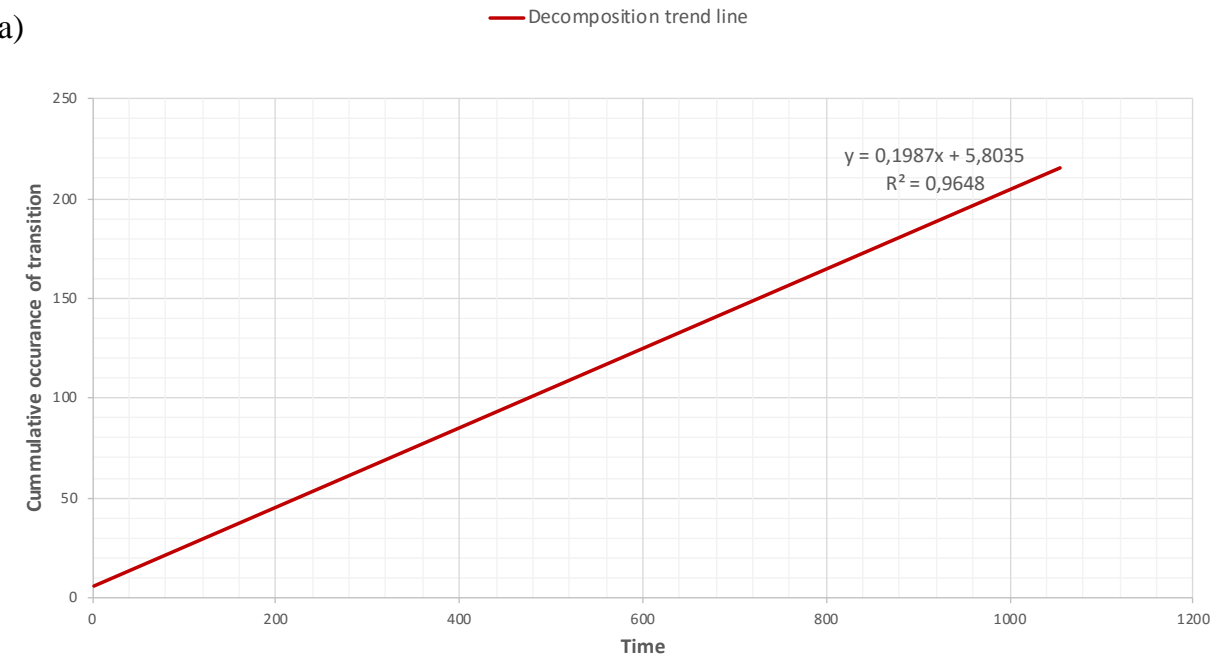

(b)

— Recomposition trend line

Figure 4. (a) Model of the cumulative occurrences for decomposition strategies, (b) model of the cumulative occurrences for recomposition strategies

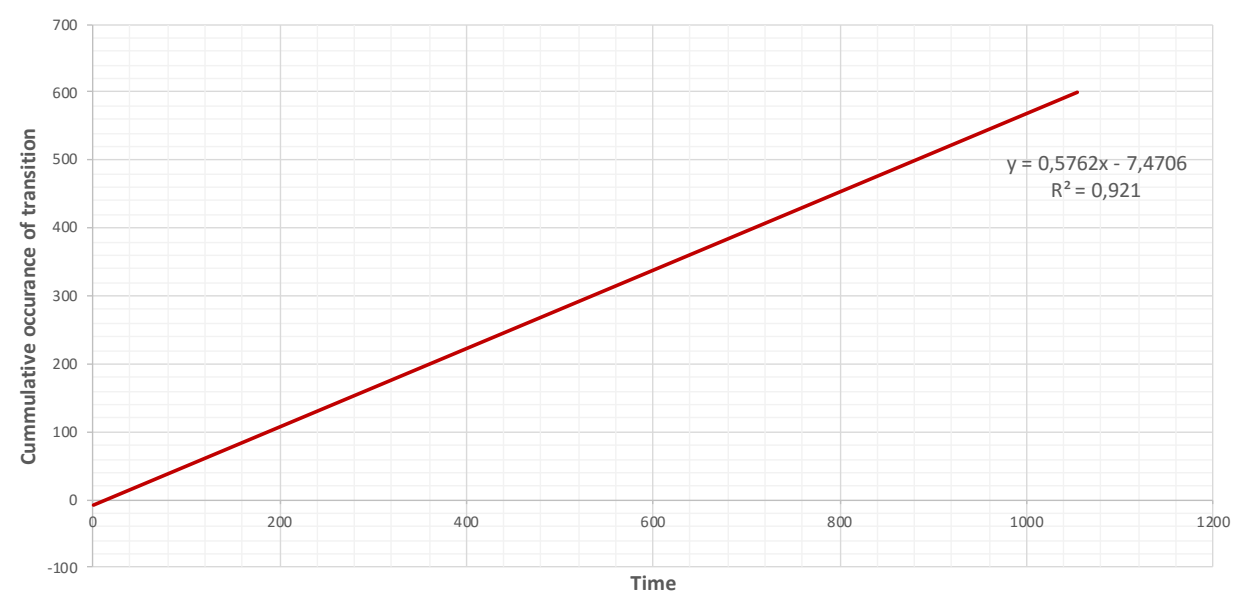

— Same system level transition trend line

Figure 5. Model of the cumulative occurrences for same system level transition 
To better understand the use of decomposition and recomposition strategies over time, we analysed the dominance of each type of strategy using a sliding window of 20 segments, representing an approximate time frame of 1 minute. To do so, we measured the ratio of the number of decomposition transitions to the number of recomposition transitions over 20 segments. A positive value implies a dominance of decomposition strategies whereas a negative value accounts for a dominance of decomposition strategies. Figure 6 represents the dominance of decomposition/recomposition strategies over time for one of the sessions. This result is common across all sessions and captures the iterative alternation of a team's cognitive effort on decomposition and recomposition strategies.

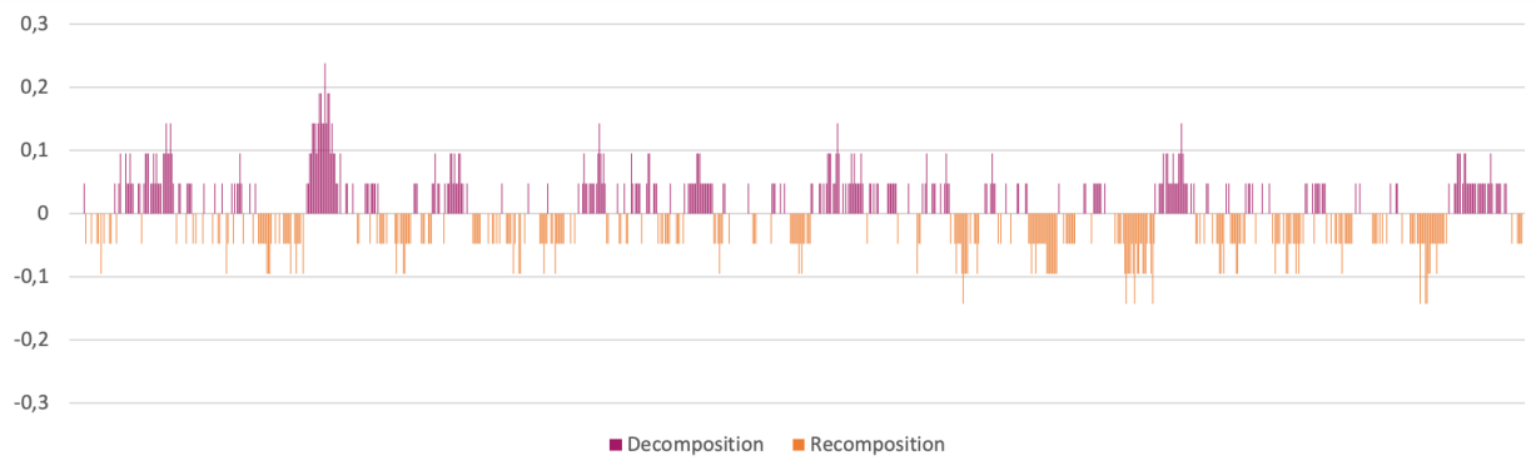

Figure 6. Timeline illustrating the dominance of a team cognitive effort overtime on decomposition or recomposition strategies

\subsection{Decomposition and recomposition strategies associated to design processes}

We explored the relationship between design processes described through the FBS ontology and system level transitions strategies. To do so, we analysed the co-occurrence of design issues transitions, forming design processes, and system level transitions, describing either a change in system level (recomposition or decomposition) or staying in the same level processes. The correspondence analysis graph in Figure 7(a) illustrates a graphic representation of the qualitative relationship between design processes and system level transitions. The FBS processes are mapped onto the three transitions in Figure 7(b).
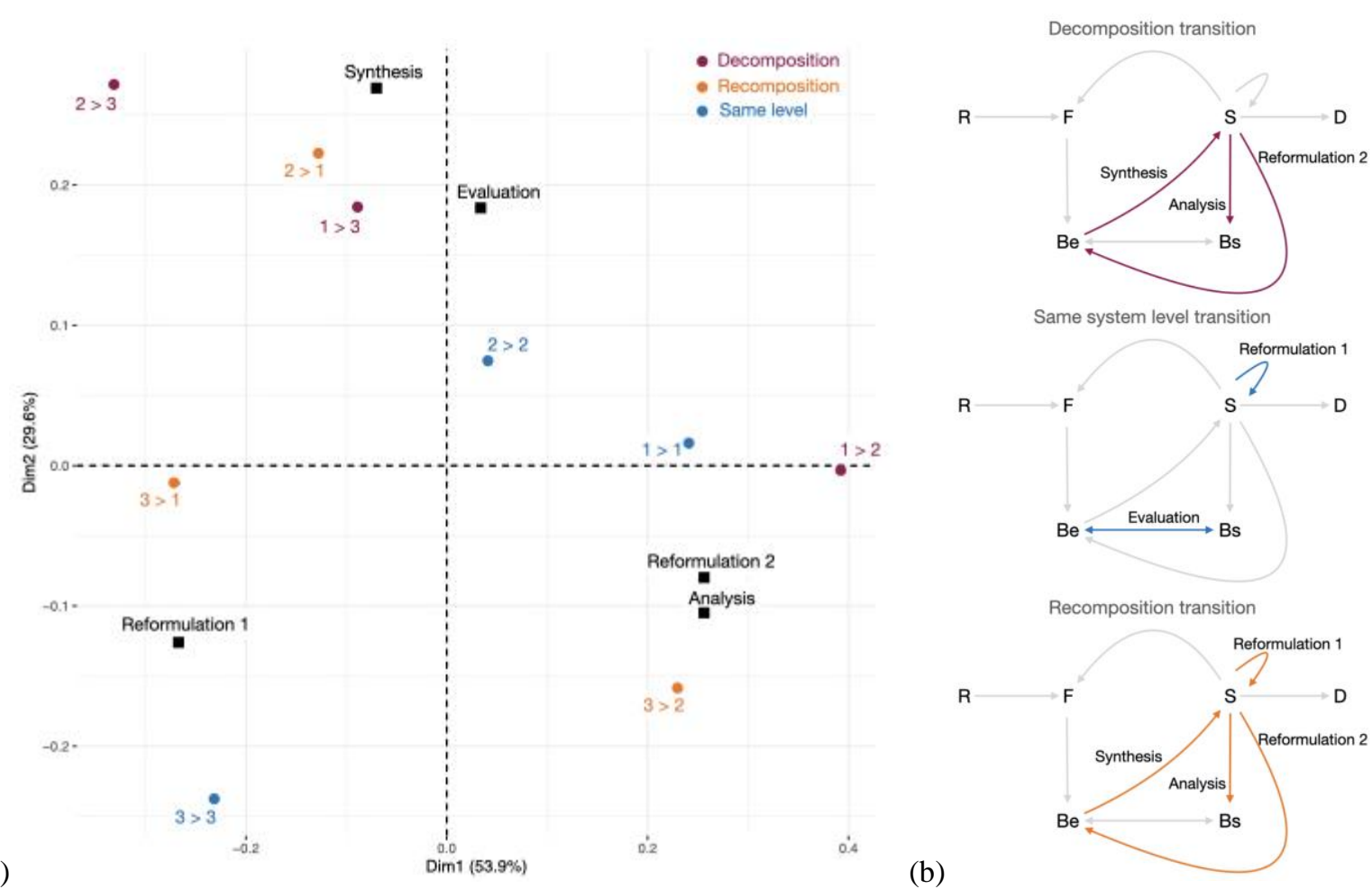

Figure 7. Relation between system level transitions and FBS design processes: (a) correspondence analysis and (b) representation of design processes on the FBS model for each decomposition/recomposition strategy 
Decomposition processes (level 1 or 2 to level 3) are associated with Synthesis as they sit in the same quadrant of the correspondence analysis graph. Similarly, decomposition from system to sub-systems (level 1 to level 2) is associated with Analysis and Reformulation 2. Recomposition processes are associated with a range of design processes: Synthesis (level 2 to level 1), Reformulation 1 (level 3 to level 1), Analysis and Reformulation 2 (level 3 to level 2). Evaluation processes are uniquely associated with same system level transitions at the system (Level 1) and sub-system levels (Level 2). Reformulation 1 relates to same system levels transition for sub-systems' details (level 3).

\section{DISCUSSION AND CONCLUSION}

The first research question tackled in this study is: What are the decomposition/recomposition strategies of professional engineering teams over time? Unexpectedly, the frequency of occurrence of decomposition and recomposition processes over time is constant. Based on theoretical frameworks (Alexander, 1964; Pahl et al., 2007; Simon, 1973) decomposition processes were expected to be higher in the first part of the session and lower towards the end. The inverse trend was expected for recomposition processes (see Figure 1). Empirical results (Gero and Song, 2017) using the same method and coding schemes pointed to a decrease of both types of strategies overtime. Our results reveal a different pattern where both decomposition and recomposition strategies occur recurrently over time (Figure 8). For all nine co-design sessions studied, each team behaved similarly in using decomposition and recomposition strategies: a fast iteration of decomposition and recomposition over time periods of 5 to 10 minutes. Such findings support the concept of an opportunistic design approach (Visser, 1994) and the co-evolution of the design/solution problem space (Dorst and Cross, 2001; Maher and Poon, 1996).

Further analysis is needed to understand if each period of decomposition/recomposition corresponds to the exploration of a specific sub-problem. To do so, we will rely on techniques from latent semantic analysis (LSA) to explore the correlation between elements designers focus on and patterns of decomposition/recomposition strategies. The unexpected results could be a signature of this company's design thinking approach. As mentioned earlier, all 27 participants were from the same company and are used to collaborating. The results presented here are part of a larger study including 10 other co-design sessions with professional engineers from another company. The analysis of these subsequent co-design sessions will provide complementary empirical data to determine whether the behaviour reported here is repeated with another cohort.

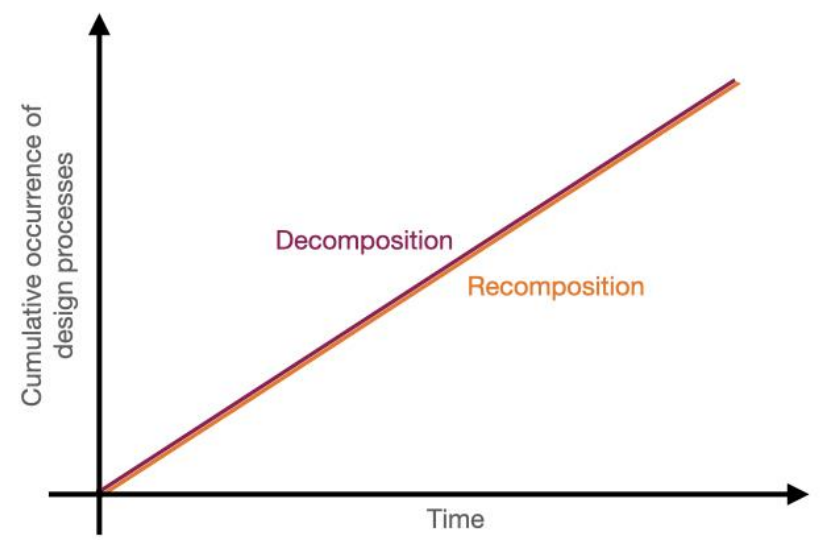

Figure 8. Temporal modelling of decomposition/recomposition strategies based on empirical results

The second research question focused on: How do decomposition and recomposition strategies correlate with design processes? Results show that similar design processes are concurrent with recomposition and decomposition strategies. Decomposition strategies were expected to correspond to analysis process. While this was found in the results, we observed that synthesis and Reformulation 2 processes were also associated to decomposition strategies. Synthesis processes occur while designers used recomposition strategies as expected. Recomposition strategies also built on Analysis, Reformulation 1 and Reformulation 2 processes. The same design process is exploited for either decomposition or recomposition strategies. For Synthesis processes, an expected Behavior (Be) at a 
sub-system level can be recomposed into a system level Structure (S) or decomposed into a subsystem detail Structure (S).

The study has some limitations that will be addressed in future work. Our findings are based on a controlled experiment, where engineers engaged in a design task outside of their natural design environment. Therefore, this study includes the inherent limitations of controlled design studies. The number of sessions analysed (9 sessions) is too small to infer general trend. Participants work in the same company, therefore our results could simply be a model of this company's design thinking approach. Our team is collecting more data from other companies to obtain more robust results.

Research presented in this paper is a first step toward understanding the underlying dynamic of decomposition and recomposition strategies of design teams. Results provide an empirical foundation to model the behaviour of engineering professionals and refine theoretical understanding of the use decomposition and recomposition strategies in design practice. These findings support that integration of team members across temporal phases of a project is relevant as decomposition and recomposition is constant overtime. To better understand the implication of such findings for team management, more research is needed, specifically exploring the connection between systems and the quality of the result in terms of creativity, innovation and performance.

\section{ACKNOWLEDGEMENTS}

This material is based upon work supported by the National Science Foundation under Grant Nos. 1761774 and 1762415 . Any opinions, findings, and conclusions or recommendations expressed in this material are those of the authors and do not necessarily reflect the views of the National Science Foundation.

\section{APPENDIX: DESIGN BRIEF}

Design Next-Generation Personal Assistant and Entertainment Systems for the year 2025

Personal Entertainment Systems (PES) is one of the most comprehensive entertainment companies in the world. In order to keep its leading position in the industry of entertainment, PES cooperates with many agents to explore the possibilities of new types of entertainment. Your design team has been invited to help in designing the next generation of a personal assistant and entertainment system suitable for family use in the year 2025.

Concept Design: In the context of engineering, a characteristic feature of the product design-related function is the description of products. Concept design includes a thorough roadmap from concept generation to production to product launch. See figure below:
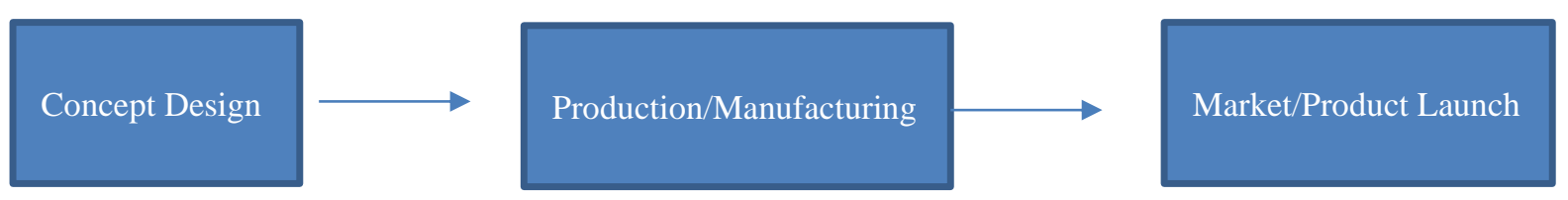

The aim of concept design is to prepare for concurrent engineering by specifying the fundamental solution to the design problem.

Task: Your team is tasked with producing concept designs of a personal assistant and entertainment system suitable for family use for the year 2025. For this project, your team should focus on: what this system would be, how this system works and interacts with people, and what the personal assistant and entertainment system would provide. Your goal is to produce a number of concepts and then develop one of those concepts into a detailed design. At the completion of the session, please present sketches (using the whiteboard) and a verbal description of your solution. Your team will have 60 minutes to complete this task.

\section{REFERENCES}

Akin, O. and Akin, C. (1996), "Frames of reference in architectural design: analysing the hyper acclamation (Ah-a-.t)", Vol. 17 No. 4, pp. 341-361.

Alexander, C. (1964), Notes on the Synthesis of Form, Harvard University Press. 
Austin-Breneman, J., Honda, T., \& Yang, M. C. (2012). A study of student design team behaviors in complex system design. Journal of Mechanical Design, 134(12), 124504. https://doi.org/10.1115/1.4007840

Ball, L.J., Evans, J., Dennis, I. and Ormerod, T.C. (1997), "Problem-solving strategies and Expertise in engineering design", Thinking \& Reasoning, Vol. 3 No. 4, pp. 247-270.

Cross, N. (1982), "Designerly ways of knowing”, Design Studies, Vol. 3 No. 4, pp. 221-227.

Dorst, K. and Cross, N. (2001), "Creativity in the design process: co-evolution of problem-solution", Design Studies, Vol. 22 No. 5, pp. 425-437.

Ericsson, K.A. and Simon, H. A. (1984), Protocol Analysis: Verbal Reports as Data, MIT Press.

Gero, J.S. (1990), "Design prototypes: a knowledge representation schema for design", AI Magazine, Vol. 11 No. 4, pp. 26-36.

Gero, J.S. and Kannengiesser, U. (2004), “The situated function-behaviour-structure framework”, Design Studies, Vol. 25 No. 4, pp. 373-391.

Gero, J.S. and Mc Neill, T. (1998), “An approach to the analysis of design protocols", Design Studies, Vol. 19 No. 1, pp. 21-61.

Gero, J.S. and Song, T. (2017), "Decomposition/Recomposition design behavior of student and professional engineers", ASEE Annual Conference \& Exposition, Columbus, Ohio, USA.

Goel, V. and Pirolli, P. (1992), "The structure of design problem spaces”, Cognitive Science, Vol. 16, pp. 395-429.

Gralla, E.L., Herrmann, J.W. and Morency, M. (2019), "Design problem decomposition: an empirical study of small teams of facility designers", Research in Engineering Design, Vol. 30 No. 2, pp. 161-185.

Guindon, R. (1990). Designing the design process: Exploiting opportunistic thoughts. Human-Computer Interaction, 5(2-3), 305-344. https://doi.org/10.1080/07370024.1990.9667157

Ho, C.-H. (2001), "Some phenomena of problem decomposition strategy for design thinking: differences between novices and experts", Design Studies, Vol. 22 No. 1, pp. 27-45.

INCOSE (2015), Systems Engineering Handbook: A Guide for System Life Cycle Processes and Activities, Fourth Edition, INCOSE-TP-2003-002-04, International Council on Systems Engineering (INCOSE), San Diego, CA.

Kahneman, D. (2011), Thinking, Fast and Slow, Penguin Books, London, UK.

Kannengiesser, U. and Gero, J.S. (2019), “Design thinking, fast and slow: A framework for Kahneman's dualsystem theory in design", Design Science, Vol. 5, p. e10.

Kannengiesser, U., and Gero, J.S. (In review), What distinguishes a model of system engineering from other models of design?

Lawson, B. (2006). How designers think: the design process demystified, Elsevier/Architectural Press.

Liikkanen, L.A. and Perttula, M. (2009), "Exploring problem decomposition in conceptual design among novice designers", Design Studies, Vol. 30 No. 1, pp. 38-59.

Maher, M.L. and Poon, J. (1996), "Modeling design exploration as co-evolution”, Computer-Aided Civil and Infrastructure Engineering, Vol. 11 No. 3, pp. 195-209.

McCracken, W. M. (1997). Portfolio assessment in design education, FIE, Atlanta, Georgia

Pahl, G., Beitz, W., Feldhusen, J. and Grote, K. (2007), Engineering Design: A Systematic Approach, 3rd ed., Springer, London.

Rittel, H. and Webber, M. (1973), "Dilemmas in a general theory of planning", Policy Science, Vol. 4, pp. 155-169.

Rowe, P.G. (1992), Design Thinking, MIT Press., Cambridge Massachussets.

Simon, H.A. (1969), The Sciences of the Artificial, Dunod.

Simon, H.A. (1973), “The structure of ill structured problems”, Artificial Intelligence, Vol. 4 No. 3-4, pp. 181-201.

Song, T., Becker, K., Gero, J., DeBerard, S., Oenardi, L. and Reeve, E. (2016), "Problem decomposition and recomposition in engineering design: A comparison of design behavior between orofessional engineers, engineering seniors, and engineering freshmen.”, Journal of Technology Education, Vol. 27 No. 2, pp. 37-56.

Sun, G., Yao, S. and Carretero, J.A. (2016), “An experimental approach to understanding design problem structuring strategies", Journal of Design Research, Vol. 14 No. 1, p. 94.

Tobias, C., Herrmann, J.W. and Gralla, E.L. (2015), "Exploring problem decomposition in design team discussions", Proceedings of the 20th International Conference on Engineering Design (ICED 15), Vol. 11:Human Behaviour in Design, Design Education, Milan, Italy, pp. 111-120.

Van Someren, M.W., Barnard, Y.F. and Sandberg, J.A.C. (1994), The Think Aloud Method: A Practical Guide to Modelling Cognitive Processes, Academic Press, London.

Visser, W. (1994), "Organisation of design activities: Opportunistic, with hierarchical episodes", Interacting with Computers, Vol. 6 No. 3, pp. 239-274. 